\title{
The Role of ICT in Reducing Maternal and Neonatal Mortality Rate in Tanzania
}

\author{
Ally S. Nyamawe \\ College of Informatics and Virtual Education, \\ The University of Dodoma \\ Dodoma, Tanzania
}

\author{
Hassan Seif \\ College of Informatics and Virtual Education, \\ The University of Dodoma \\ Dodoma, Tanzania
}

\begin{abstract}
Tanzania is striving to achieve Millennium Development Goals (MDG) 4 and 5 that call for reducing Neonatal and Maternal Mortality Rate (MNMR) by 2015. In 2010, Neonatal Mortality Rate (NMR) was recorded at 51 per 1,000 live births and Maternal Mortality Rate (MMR) at 454 per 100,000 live births. Despite of the devoted efforts and significant progress, still the mortality rate needs attention and effective intervention to cut it down considerably. The capability and power of Information and Communication Technology (ICT) need to be effectively exploited in revolutionizing health sector in Tanzania. The witnessed expansion of mobile phones usage and extended network coverage presents potential opportunity in enhancing quality of Maternal, Neonatal and Child Health (MNCH) services delivery. This paper explores the role of ICT in reducing MMR and NMR, existing solutions are intensely reviewed to discover what is lacking and provide necessary recommendations.
\end{abstract}

\section{General Terms}

ICT for Health

\section{Keywords}

ICT, Maternal, Mortality Rate, Neonatal.

\section{INTRODUCTION}

Tanzania is one of the ten countries contributing to $61 \%$ and $66 \%$ of the global total of maternal and newborn deaths, respectively [1]. The health of the mother is mostly closed to the health and survival of the child. The Improving of Maternal Newborn and Child Health, is the progress of MDG 4 and 5. Hence there is a need of finding the way of achieving these two MDGs that relate to women, Newborn and children. Most African countries are on the whole off tract to achieving the MDGs 4 and 5 for maternal, newborn and child health by 2015 [2]. Reducing Maternal, newborn and child mortality in Africa is a challenge of the New Millennium. Each year in Sub Saharan Africa 279,000 women die due to complications of pregnancy and another 4.5 million children die before their first birthday; 1.2 million of them die in the first month of life [2].

Neo-natal mortality is the death of a child who is born alive, but dies within the first 28 days of life. It can be measured in terms of neo-natal mortality rate, which is the number of deaths per 1,000 live births [3]. In Tanzania somehow there has been significant progress in the reduction of neonatal mortality rates. Infant mortality rate has declined from $68 / 1,000$ live births in $2004 / 05$ to 58 per 1,000 live births in $2007 / 08$ and then to $51 / 1,000$ in 2009/10 [4]. Maternal mortality is the death of a woman while pregnant, or within 42 days of termination of pregnancy, or its management, but not from accidental or incidental causes. It can be measured in terms of maternal mortality ratio, which is the number of maternal deaths during a given period per 100,000 live births [3]. The 2009/10 Demographic Health survey (DHS) results show that the maternal mortality ratio during the ten-year period is 454 maternal deaths per 100,000 live births compared to 529/100,000 (1996) and 578/100,000 (2004/05) maternal death [4].

Neonatal conditions like birth asphyxia and infections are the major causes of death in young children, followed by pneumonia, diarrhoea and malaria. AIDS is also a major killer, responsible for about 9 per cent of under-five deaths [5]. Furthermore, around 32 per cent of all under-five deaths occur in the first 28 days of life - many infants survive for only a few days [5]. Neonatal deaths are inextricably linked to the health of the mother during pregnancy and to the conditions of delivery and newborn care. Poor quality of care due to an insufficient number of skilled health workers and lack of basic equipment, as well as long distances from home to health care facilities are major deterrents to facility delivery. Women living in rural areas, those who come from the poorest families and those who are less educated, have the least access to skilled attendance at delivery. Women who start having children in adolescence tend to have more children and shorter spacing between pregnancies - all of which are risk factors for maternal and neonatal mortality. The neonatal mortality rate is highest among mothers under20 years of age at 45 per 1000 live births compared with 29 per 1000 for mothers aged 20 to 29 years [5].

Ssewanyana et al. [6] Noted that, most infant deaths result from a combination of preventable or treatable diseases such as: Pneumonia, Diarrhoea, and Malaria and immunizable diseases such as Tuberculosis and Measles.

Moreover, maternal deaths are caused mainly by the factors attributable to health complications during pregnancy and delivery. Newborn deaths are as well caused by numerous factors and mostly occur in the first week of life. The three main causes of neonatal death in Tanzania are well known: $32 \%$ die from infections; $27 \%$ die from complications of preterm birth; $26 \%$ die from birth asphyxia [7].

In addition to that, one of the major factors accelerating to high Maternal Mortality Rate (MMR) is a failure for a pregnant woman from accessing health facility promptly. This delay is grouped into three major phases, namely; (i) Recognizing the need for health care and in the decisionmaking process; (ii)Arrival at a health facility; and (iii) Receiving appropriate and adequate care at the health facility [8]. Underlying determinants that cause the delays are the 
position of women in society, large geographical distances, weak health systems, poverty and lack of education [8].

\section{RELATED WORKS}

With the advancement of ICT in recent world, a number of ICT based solutions can be explored in the effort to improve health care. MDG8 calls upon the exploitation of benefits of the existing new technologies, especially those related to information and communication [8]. As MDG 8 suggests, the authors also see potentiality of employing ICT in addressing $\mathrm{MNCH}$ care. ICTs present a large, unexploited potential for transforming governance and transparency in the health sector in Africa to achieve "more health for money spent" and thereby improve the efficiency of health spending, both domestic and donor-financed [9].

\subsection{The use of Mobile Phones in bettering Maternal and Neonatal Health (MNH)}

In the modern era, there has been a significant expansion and coverage of mobile network. In Tanzania mobile phones usage and network coverage has rocketed at a very surprising rate. Tanzania Communications Regulatory Authority (TCRA) estimates that $75-80 \%$ of the population has access to a mobile phone. Expanding mobile phone penetration and network coverage can remove traditional geographic and economic barriers to health care, particularly in emergency situations that can be life threatening, as is often the case for MNH [10]. Mobile technologies can be efficiently exploited in delivering health care services. Health information can be instantly communicated via mobile phones as text messages [11]. According to the International Telecommunications Union (ITU) there are more than 3.3 billion mobile subscriptions worldwide. $68 \%$ of those subscriptions are in developing countries and even people living on USD 1 per day increasingly have access to mobile phones [12]. A wide range of social and economic goals can be advanced by extending the benefits of mobile phone ownership to more women. Mobile phones connect people and provide them with information that is useful in their daily lives [13]

Wired-mothers, project that employed the use of mobile phones to improve maternal and neonatal deaths in Zanzibar. Wired mothers are pregnant women linked to a primary health care unit through use of mobile phones receiving standard Short Messaging Services (SMS) reminders for care appointments and who can call the primary provider in case of acute or non-acute problems [12]. The setting of this project put the pregnant women and Traditional Birth Attendants (TBAs) at the same level as both are in the same community. As TBAs considered being closer to pregnant women, they can offer necessary assistance in case of emergency. Moreover, the channel of communication links the community to the referral level via Primary Health Care Unit (PHCU). In addition to that, the antenatal care attendance though mobile phones intervention was made. The wired-mothers' mobile phone intervention significantly increased the proportion of women receiving health care during pregnancy and after delivery. Mobile phones applications showed positive results towards improving quality maternal and newborn health care [14].

In Tanzania, there have been a number of extensive initiatives in developing mobile applications for providing pregnant women with health tips for the wellbeing of their own pregnancy and safety delivery. Expectant and new mothers receive SMSs for reminding them to visit clinics regularly and as well useful information relating to each and every phase of their pregnancy [15].
Aligned to that, Mobile Alliance for Maternal Action (MAMA) in collaboration with other development partners launched a mobile based application "Healthy Pregnancy, Healthy baby text messaging service" in Tanzania. The project aimed at delivering high quality antenatal care (ANC) information during pregnancy and 16 weeks after delivery. Information included proper nutrition and care and as well as signs of health complications. To ensure technical relevance, the "Healthy pregnancy, Healthy baby text messaging service" text messages were reviewed in 2011 by the Tanzania Ministry of Health and Social Welfare (MoHSW) through its Wazazi Nipendeni Campaign material review. Among the public health objectives that the project was expected to meet, is reduction of MMR and NMR. The Partnership has compelled anecdotal evidence that suggests that the service assists in achieving these important public health goals [16].

In March 2013, D-tree International and World Vision Tanzania started to develop and deploy a mobile phone based tool for Community Health Workers (CHWs) in Singida districts. The tool aimed at assisting CHWs in delivering preventive care and proactively identifies threatening indications and leads CHWs on what to do. Pregnant women were required to register and CHWs monitor their progress through to delivery. During the monitoring process, the tool also provides CHWs with a checklist of danger signs for the mother (during pregnancy, labor, and postpartum) and newborn, and gives instructions on how to counsel a woman regarding antenatal clinic visits, facility based deliveries, and postnatal clinic visits [17]. The solution seemed to deliver positive results.

Furthermore, Malawi implements the Information and Communications Technology for Maternal, Newborn and Child Health (ICT for MNCH) Pilot Project in Balaka District, in southern Malawi, where its hotline and tips and reminder services are together known as Chipatala Cha $\mathrm{Pa}$ Foni (CCPF), or "Health Center by Phone." It aimed to address barriers to $\mathrm{MNCH}$ information and improve the coverage and quality of $\mathrm{MNCH}$ services by; (i) increasing knowledge of facility and home-based maternal, newborn and child health $(\mathrm{MNCH})$ practices, (ii) increasing use of facility and home-based MNCH practices, and (iii) increasing client satisfaction with $\mathrm{MNCH}$ facility services [18].

Moreover there are some new apps developed suited to combating maternal and child health in Nigeria, like the mobile ultrasound app developed by the Mobisante team; the smartphone based quantitative diagnostic solution by i-calQ, and the recently developed anaemia detecting app [19].

In Tanzania majority of people have access to mobile phones, and the coverage of the cellular network is largely extended in most areas. The mobility and portability of mobile phones make it the closest mode of communication. Therefore these advantages can be exploited in bettering MNH.

\subsection{The use of website in Bettering MNH}

In Ghana they have tried to use website to address the country's Maternal and Neonatal mortality crisis. MamaYe which is an 'Akan' word meaning (Mother is Good), recently launched its website -www.mamaye.org.gh - a digital platform for maternal and newborn health stakeholders to be educated, empowered, and united in their efforts [20]. This is relevant approach; since website is considered powerful in delivering vast amount of information at per. Presentation and illustration of more lively information can easily be conveyed through website. Moreover, website can be displayed in 
different platforms including; some of mobile phones and computers. Through daily information updating on website, people can constantly build up their body of knowledge regarding $\mathrm{MNH}$, this would have positive impact in reducing MNMR.

\subsection{Some Existing ICT Tools}

Some ICT tools and open source platforms exist already that can accelerate the deployment of innovative ICT solutions for reproductive, maternal, neonatal, and child health ( $\mathrm{RMNCH})$ [21]. These tools include, but are not limited to CommCare, Medic Mobile, RapidSMS, NOKIA Data Gathering tool, Magpi (formerly EpiSurveyor), eMOCA, and the MoTech suite. Those platforms and tools have already shown promising results in gathering, collecting, and exchanging health information, as well as in delivering many communitybased healthcare services [21]. Hence by means of using and modifying some of these tools it can result into innovative ICT solution which will reduce maternal and neonatal mortality rate considerably.

\section{RESULTS AND DISCUSSION}

Maternal and Neonatal mortality has yet received exceptional considerations from national level to global. Different approaches with distinct settings have been employed to rescue the situation. ICT has been considered to be effective in reducing MMR and NMR. Numerous studies have declared the potentiality of ICT in delivering quality health services. A number of projects are exploiting the advantages of mobile phones in delivering $\mathrm{MNCH}$. Mobile phones are reasonably cheap, high mobility and widely used due to extended network coverage available today. Mobile phones to a large extent have facilitated communication across urban, rural and marginalized areas.

Delays, lack of education and lack of skilled clinic attendants are mentioned to be among the contributors of maternal and neonatal deaths. In Tanzania, the undertaken projects so far have tried to bridge the gap between expectant mothers, traditional births attendants (TBAs) and health facilities through mobile phones' services (Calling and Short messaging). Expectant mothers can constantly be linked to a health facility by receiving reminders on clinic visiting through SMSs and call a health facility for an emergency help. TBAs being closer to expectant mothers can also provide necessary support while in the effort to reach a health facility. This is expected to cut down delays significantly.

Through SMSs expectant and new mothers are able to receive information on the progress of their pregnancy and even warnings in case of complications. Mothers get informed throughout prenatal and postnatal periods. In the same line, local clinic attendants can call referral hospitals for advice in case of difficulties or challenging situations. Web portals and other ICT tools have also been extensively used to supplement delivering of information to mothers.

With the existing interventions, the mortality trend is improving towards achieving MDG 4 and 5. It is therefore expected that, if ICT- based interventions are extensively used, the mortality trend will keep improving significantly. Table 1 illustrates maternal and neonatal mortality trends from 1996 to 2010. This is depicted further in Figure 1 and Figure 2.
Table 1: Maternal and Neonatal Mortality Trends

\begin{tabular}{|c|c|c|c|c|c|c|c|}
\cline { 2 - 8 } & \multicolumn{3}{c|}{$\begin{array}{c}\text { Maternal mortality trends } \\
(\mathbf{1 0 0 , 0 0 0})\end{array}$} & \multicolumn{5}{c|}{ Neonatal mortality trends (/1000) } \\
\hline YEAR & 1996 & 2005 & 2010 & 1996 & $2004-05$ & $2007-08$ & 2010 \\
\hline TREND & 529 & 578 & 454 & 88 & 68 & 58 & 51 \\
\hline
\end{tabular}

Source: TANZANIA Country Report on the Millennium Development Goals 2010.

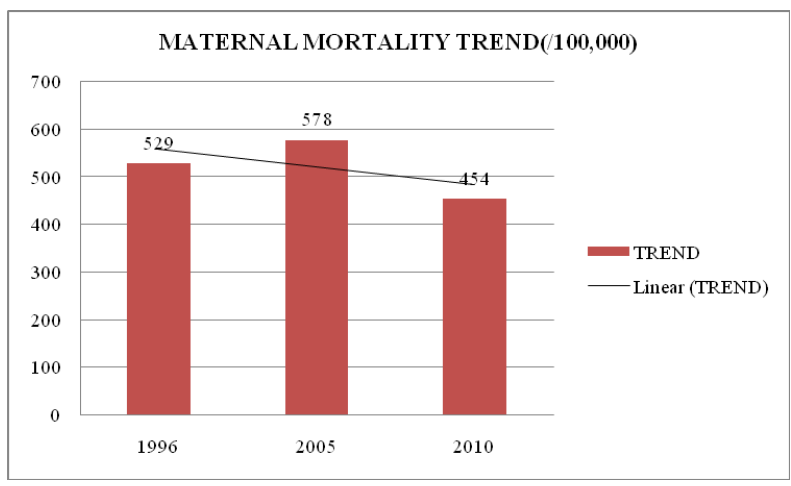

Figure 1: Maternal Mortality Trend

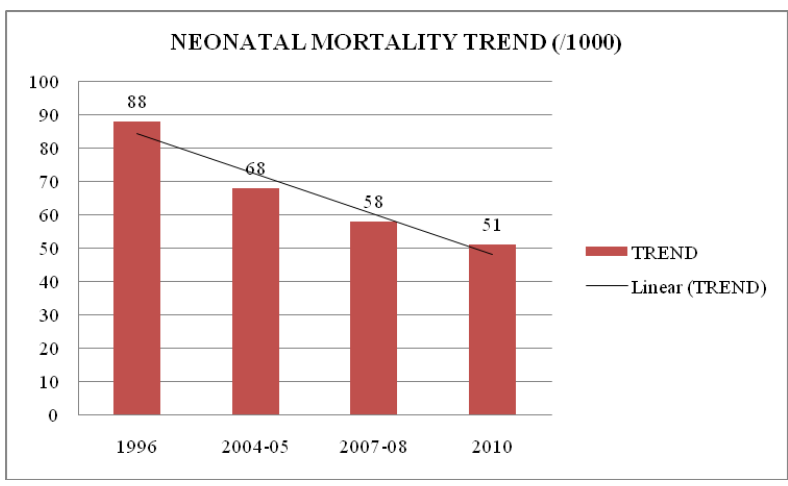

Figure 2: Neonatal Mortality Trend

To fully extend the benefits of ICT in delivering quality health services in Tanzania, there should be sustainable solutions and friendly supporting environment across the country. The mHealth technologies should be deployed entirely instead of only to some regions. The government should stress on public awareness so that majority can subscribe to mHealth services. To supplement mhealth, Web portals can be employed which will provide more information that cannot be conveyed through mobile phones concerning maternal and neonatal health care.

\section{CONCLUSION}

It needs joint efforts to achieve MDG 4 and 5. Collaboration between multiple stakeholders from ICT and health sectors can be a proper way in a battle against MMR and NMR in order to achieve MDG 4 and 5. ICT intervention in delivering quality $\mathrm{MNCH}$ care has demonstrated promising results. In Tanzania, the employed solutions need to be extended to cover large group as much as possible. To significant escalate sustainability and productivity of mHealth and related efforts, the government should institute favorable policies. To prepare tomorrow's healthy mothers, information delivering should go beyond maternal. Matured boys and girls should also be considered as potential target in receiving preparatory information to build up their body of knowledge in advance on $\mathrm{MNCH}$ care. 


\section{REFERENCES}

[1]. The National Road Map Strategic Plan to Accelerate Reduction of Maternal, Newborn and Child Deaths in Tanzania 2008 - 2015; 2008.

[2]. Mwaikambo, E. "IMPROVING MATERNAL, NEWBORN AND CHILD HEALTH IN TANZANIA: FROM SCIENCE TO ACTION", THIRD PROFESSOR HUBERT KAIRUKI MEMORIAL LECTURE, February 2010 .

[3]. Alliance For Reproductive Health Rights (ARHR) Retrieved from (http://www.mamaye.org/sites/default/files/mamaye-ghfactsheet-web_0.pdf), on $20^{\text {th }}$ April 20, 2014.

[4]. TANZANIA Country Report on the Millennium Development Goals 2010, SEPTEMBER 2011.

[5]. Maternal and child health. Retrieved from ( http://www.unicef.org/tanzania/6906.html), on $13^{\text {th }}$ April 2014.

[6]. Ssewanyana, S. and Younger, S.D. 2005 "Infant Mortality in Uganda: Determinants, Trends, and the Millennium Development Goals,". Retrieved from (http://www.saga.cornell.edu/images/wp186.pdf), on $20^{\text {th }}$ April 2014.

[7]. Situation analysis of newborn health in Tanzania. Current situation, existing plans and strategic next steps for newborn health: 2009.

[8]. A. Camielle Noordam, Barbara M. Kuepper, Jelle, S. and Anneli M. "Improvement of maternal health services through the use of mobile phones", Tropical Medicine \& International Health, Volume 16, Issue 5, pp 622-626, 2011.

[9]. Meera, S. and Kate O. 2012. ICT for Health in Africa.

[10].mHealth Alliance, "Leveraging Mobile Technologies to Promote Maternal \& Newborn Health: The current landscape \& opportunities for advancement in lowresource settings". Retrieved from (www.mhealthalliance.org), on $15^{\text {th }}$ April 2014.

[11].L'Engle, K. and Vadhat, H. "Mobile Phone Interventions for Reproductive Health (m4RH): Testing the Feasibility of Text Messaging to Improve Family Planning".
[12]. Wired mothers - use of mobile phones to improve maternal and neonatal health in Zanzibar. Retrieved from (www.enrecahealth.dk/archive), on 12 ${ }^{\text {th }}$ April 2014.

[13]. GSMA Development Fund, Cherie Blair Foundation for Women (2010).Women \& Mobile: A Global Opportunity A study on the mobile phone gender gap in low and middle-income countries. Retrieved from (http://www.gsma.com/mobilefordevelopment/wpcontent/uploads/2013/01/GSMA_Women_and_Mobile A_Global_Opportunity.pdf).

[14].Lund, S., Nielsen, Birgitte B., Hemed, M., Boas Ida M., Said, A., Said, K., Makungu, Mkoko H. and Rasch, V. Mobile phones improve antenatal care attendance in Zanzibar: a cluster randomized controlled trial, 2014.

[15]. Better care for unborn babies in Tanzania due to SMS Retrieved from (http://www.texttochange.org/news/better-care-unbornbabies-tanzania-due-sms), on $16^{\text {th }}$ April 2014.

[16].Mobile Alliance for Maternal Action, Spotlight July 2013. Retrieved from (http://mobilemamaalliance.org/sites/default/files/1749MAMA-Spotlight-July-v4-TC\%5B2\%5D.pdf).

[17].WORLD VISION'S GLOBAL MHEALTH PORTFOLIO. Retrieved from (http://www.wvi.org/health/mhealth-tanzania), on $16^{\text {th }}$ April 2014.

[18].Watkins, C.S., Robinson, A. and Dalious, M., 2013 "Evaluation of the Information and Communications Technology for Maternal, Newborn and Child Health Project Known locally as "Chipatala Cha Pa Foni" (Health Center by Phone)", Invest in Knowledge Initiative (IKI)

[19].Owobu, E. 2013 App Development in Combating Maternal and Child Health Mortality in Developing Countries. Retrieved from (http://www.techph.org/appdevelopment-in-combating-maternal-and-child-healthmortality-in-developing-countries/), on $20^{\text {th }}$ April 2014

[20].Using ICT Tools to address Ghana's Maternal and Neonatal Crisis. Retrieved from (http://thechronicle.com.gh/using-ict-tools-to-addressghanas-maternal-and-neonatal-crisis/), on $20^{\text {th }}$ April 2014.

[21].ITU, 2013. I C T f o r I m p r o v i n g Information and Accountability for Women's and Children's Health. 